\title{
Neutrino Spectroscopy of the Early Phase of Nearby Supernovae
}

\author{
F. K. Sutaria*, A. Ray*† \\ * Theoretical Astrophysics Group, Tata Institute of Fundamental Research, Bombay 400 005, India \\ ${ }^{\dagger}$ Laboratory for High Energy Astrophysics, Goddard Space Flight Center, Greenbelt, MD 20771 \\ E-mail: fks@dashoo.tifr.res.in,akr@tifrvax.tifr.res.in
}

(Accepted for publication in Phys. Rev. Lett. on 11th Jul., 1997)

\begin{abstract}
Neutrinos emitted during stellar core collapse up to their trapping phase carry information about the stage from which the Supernova explosion process initiates. The dominant $\nu_{e}$ emission mechanism is by electron capture on free protons and f-p shell nuclei and the spectrum of these neutrinos is a function of the ambient physical conditions within the core as well as the nuclear equation of state. The number of collapse phase $\nu_{e}$ which can be detected by Super-Kamioka and Sudbury Neutrino Observatory from a Supernova within $1 \mathrm{kpc}$, and their generic energy spectra are given.
\end{abstract}

Ms number $\quad$ PACS number: 97.60.Bw, 95.55.Vj, 26.50.+x, 95.85.Ry 


\section{INTRODUCTION}

Although several dozen Supernovae are routinely detected in external galaxies every year in the optical channel, the small interaction cross-section of neutrinos with matter together with the enormous dilution of their flux due to large distance of external galaxies has prevented the detection of Supernovae in the neutrino channel with the exception of SN1987A. However, since large experiments with the main purpose of detecting Solar neutrinos are either already on line (such as Super-Kamioka (SK)) or expected to become operational soon (e.g. Sudbury Neutrino Observatory (SNO)) it is pertinent to consider what such experiments can reveal about late stages of stellar evolution, such as the gravitational collapse of a massive star leading to a Supernova - should such an event occur in our galaxy close to the Earth 叫, 设]

The 19 neutrinos detected from SN1987A were most likely to have been emitted during the post-bounce phase as their total fluence during the proto neutron star cooling phase (at $\simeq 10^{58}$ ) is much larger than that during the collapse phase $\left(\simeq 10^{56}\right)$. Much of the information pertaining to the conditions in which the neutrinos are originally produced, such as the nuclear and thermodynamic properties of the core of the Supernova are altered because these neutrinos undergo inelastic scattering with the overlying stellar matter in the post neutrino trapping phase. Neutrinos which are emitted through electron captures on the nuclei present in the pre-supernova and collapsing core before it reaches neutrino trapping density $\left(\simeq 10^{12} \mathrm{gm} / \mathrm{cm}^{3}\right)$ [ $]$, however, stream freely through the stellar matter without any further interactions. These pre-trapping neutrinos carry with them information on both the physical conditions within the core, as well as it's nuclear configuration e.g. the ratio of the number density of free protons to that of heavy nuclei. The last quantity can be dependent on the nuclear equation of state relevant to this region of collapse. Since neutrinos act as probes of the dynamic, thermodynamic and nuclear properties of the presupernova and collapsing core, their detection and measurement of energy spectrum can have significant implications. The time evolution of the detected spectrum could also reveal the dynamical timescale - a clue to the average density and mass of the stellar core which may have implications for black hole vs neutron star formation. The collapse phase emission is in the $\nu_{e}$ channel. Since the $\nu_{\mu}$ and $\nu_{\tau}$ flavors can be detected only by neutral current (n.c.) reaction on $d$ nuclei in SNO or by $\nu-e^{-}$scattering, any excess of neutrinos detected in the n.c. channel (over the value measured from the charged current (c.c) channel) in SNO would be indicative of neutrino oscillation. The extent of the reduction of lepton fraction during stellar collapse has important implications for shock formation stages and the overall dynamics - even in the delayed explosion stage, since it determines, through the original energy of the bounce shock, and the entropy profile in the outer core, the position of the stalled shock [5].

\section{NUMBER OF NEUTRINOS EMITTED AND DETECTED}

Since electron captures take place throughout the mass of the iron core, the fluence of $\nu_{e}$ received per unit area at a distance D up to a certain stage characterized by the massweighted electron fraction $\left(\mathrm{Y}_{e f}\right)$ in the core, is given by $F_{\nu_{e}}=M_{C} \Delta Y_{e} /\left(4 \pi D^{2} m_{B}\right)$, where $M_{C}$ is the mass of the collapsing "Fe" core, $\mathrm{m}_{B}$ the baryonic mass, and $\Delta Y_{e}=Y_{e_{f}}-Y_{e_{i}}$ 
is the change in the lepton fraction from the "start" of collapse (corresponding to the end of Kelvin-Helmholtz contraction following Si exhaustion) to the point where $\nu_{e}$ begin to be trapped and scattered by overlying matter in the collapsing core. Stellar core density of $\approx 2 \times 10^{11} \mathrm{~g} / \mathrm{cm}^{3}$ is still transparent for 10 to $20 \mathrm{MeV}$ neutrinos. The spectrally integrated fluence of $\nu_{e}$ at a distance of $1 \mathrm{kpc}$, as $Y_{e}$ changes from 0.42 to 0.39 in a $1.4 \mathrm{M}_{\odot}$ stellar core (of a $15 \mathrm{M}_{\odot}$ star) is: $F_{\nu}=4.2 \times 10^{11} \mathrm{~cm}^{-2}$. The energy of the infall neutrino burst up to this stage is: $E_{\nu_{e}}=7.2 \times 10^{50} \mathrm{erg}$.

The flux, direction and the spectra of the neutrinos can be measured by the charge current dissociation of the deuterium nucleus $\left(\nu_{e}(d, p p) e^{-}\right)$in the Sudbury Neutrino Observatory (SNO) w1 with a fiducial mass of $1 \mathrm{Kt}$ of high purity $\mathrm{D}_{2} \mathrm{O}$. It is also possible to detect $\nu_{e}$ and obtain spectral information by means of the neutrino-electron scattering reaction $\left(\nu_{e}+e^{-} \rightarrow \nu_{e}+e^{-}\right)$in SNO as well as in the light water detector Super Kamioka. Apart from the reactions mentioned above which can perform spectroscopy (i.e. measure the incoming neutrino energy), the neutral current dissociation of $d$ by the reaction $\nu_{e}(d, p n) \nu_{e}$ can obtain the the total neutrino flux (of all flavors), but since it does not give any neutrino energy information, it is equivalent to neutrino "photometry".

The number of $\nu_{e}$ events which can be expected to be detected in the SNO detector through neutrino-induced c.c. reaction on the target $d$ nuclei is given by: $n_{\nu_{e}}=F_{\nu} \sigma_{c c}\left(\epsilon_{\nu_{e}}\right) N_{d}$ where $N_{d}\left(=6.02 \times 10^{31}\right)$ is the total number of target nuclei present in the $1 \mathrm{Kt}$ detector. The charge current and neutral current cross-sections $\left(\sigma_{c c}\left(\epsilon_{\nu_{e}}\right)\right.$ and $\sigma_{n c}\left(\epsilon_{\nu_{x}}\right)$ respectively), have been computed for the $\nu$ - $d$ process by Bahcall et al. [6] and accurate fits to these crosssections between 5 to $40 \mathrm{MeV}$ are given as $\downarrow$ : $\sigma_{i}=\alpha_{i}\left(\epsilon_{\nu}-\epsilon_{t h, i}\right)^{2.3}$ where $i=c c$ and $n c$, $\alpha_{c c}=1.7 \times 10^{-44} \mathrm{~cm}^{2}, \alpha_{n c}=0.85 \times 10^{-44} \mathrm{~cm}^{2}, \epsilon_{t h, c c}=2.2 \mathrm{MeV}$ and $\epsilon_{t h, n c}=1.44 \mathrm{MeV}$.

For the $\mathrm{H}_{2} \mathrm{O}$ based Cerenkov detector (Super-Kamioka) the $\left(\nu_{e}, e^{-}\right)$scattering events would be the main source of $\nu_{e}$ spectral information since the corresponding energy thresholds for charge current and neutral current interactions for ordinary water are much higher. (During the collapse stage, the neutrino flux is almost entirely in neutrinos of the electron type; anti-neutrinos of various kinds, as well as neutrinos of the mu or tau type are generated in copious numbers only in the hot post core bounce phase). The relevant $\left(\nu_{e}, e^{-}\right)$scattering cross-section is 12] : $\sigma_{e}=(1 / 2)\left(4 G^{2} m_{e}^{2} \hbar^{2} / \pi c^{2}\right)(7 / 12)\left(\epsilon_{\nu} / m_{e} c^{2}\right)$

The number of detections by Super-Kamioka (mass $32 \mathrm{kt}$ ) and SNO for a supernova explosion $1 \mathrm{kpc}$ away, for several possible scenarios of stellar core collapse are reported in Table 1. The $15 \mathrm{M}_{\odot}$ star collapse is initiated from thermodynamic conditions as in [8] ( $\mathrm{Y}_{e i}$ $\left.=0.420, S_{i} / k_{B}=1.00, \mathrm{~T}_{i}=0.7178, \rho_{10}=0.37\right)$, while the $25 \mathrm{M}_{\odot}$ star's single zone initial conditions are similarly derived from the data reported in [15] and an expression for the core averaged entropy $\left(\mathrm{Y}_{e i}=0.423, S_{i} / k_{B}=1.14, \mathrm{~T}_{i}=0.6756, \rho_{10}=0.15\right)$.

\section{INCIDENT NEUTRINO SPECTRUM:}

To estimate the number of neutrinos incident upon an underground detector, at a distance $D$ kpc from the SN, we use the stellar core collapse code developed in ref. [7]. This code uses the "single-zone" method outlined in [9] (hereafter BBAL) exploiting the homology of the core structure during collapse but used the electron capture rates for both heavy nuclei and free protons with temperature dependent phase space factors and neutron shell-blocking in nuclei included where relevant, an analytic equation of state for hot dense 
matter as in [8] and a treatment of the neutrino trapping and leakage at densities above a few times $10^{11} \mathrm{~g} / \mathrm{cm}^{3}$. The core of a massive star collapses under its own gravity when the pressure support from degenerate electrons is reduced through the capture of electrons in the stellar material. The electron capture on neutron rich heavy nuclei initially proceeds primarily through the allowed type $(\Delta l=0)$ Gamow Teller transitions. As core density exceeds $\simeq 10^{11} \mathrm{gm} / \mathrm{cm}^{3}$, the nuclei become become more and more massive and too neutron rich to allow $\mathrm{e}^{-}$-capture to take place through allowed Gamow-Teller transitions from the ground state. This is because the allowed states for $p$ to $n$ transition within the nucleus are already filled by the neutrons (neutrons shell blocked) and the transition strength for typical captures like ${ }^{56} \mathrm{Fe} \rightarrow{ }^{56} \mathrm{Mn}$ used earlier (as in [9]) is no longer representative of typical nuclear $\mathrm{e}^{-}$-capture rates. It was estimated [8] that the effective transition strength for electron capture under these circumstances due to first forbidden transitions and due to thermally unblocked allowed transitions. However it was shown [13], 14 that the dominant unique first forbidden transition strength was actually negligible compared to the thermally unblocked strength under the typical core collapse conditions. Therefore, after neutron-shell blocking sets in, (when $(\mathrm{A}, \mathrm{Z})>{ }^{74} \mathrm{Ge}$ ) the sum rule for the Gamow Teller transition operator $\left|M_{G T}\right|^{2}$ decreases from a typical value of 2.5 [6] to about 0.1 . The $\mathrm{e}^{-}$-capture rate on a single nucleus $X(A, Z)$ in the initial state $i$ to the final state $\mathrm{j}$ is given by:

$$
\lambda_{i j}=\ln 2 \frac{f_{i}\left(T, \mu_{e}, Q_{i j}\right)}{f t_{i j}}
$$

where $f t_{i j}$ is related to $\left|M_{G T}\right|^{2}$ by $f t_{i j}=3.596-\log \left|M_{G T}\right|^{2}$ for allowed Gamow-Teller type transitions (for free protons, $\left.\log \mathrm{ft}_{f . p .}=3.035\right)$. The factor $f_{i}\left(T, \mu_{e}, Q_{i j}\right)$ is the phase space factor for the allowed transition, which is a function of the ambient temperature $T$, the Fermi-energy of the electron $\mu_{e}$ and the Q-value for the reaction $Q_{i j}$. ¿From energy conservation, the neutrino energy is $E_{\nu_{e}}=E_{e}-Q_{i j}$.

In the calculations performed here, we follow the self-similar density evolution of a single zone which is represents the properties of the polytropic distribution of matter in the core and, in which the density, entropy per nucleon, and electron fraction are allowed to evolve self-consistently with the electron capture physics and the consequent changes in nuclear and thermodynamic variables. During the collapse, a change in entropy controls the fraction of the dripped protons with respect to that of the heavy nuclei, and as the spectrum of neutrinos generated by electron capture on protons are different from captures on heavy nuclei, this influences the overall neutrino spectrum received on earth. The received neutrino spectrum may depend not only upon the initial conditions from which the collapse started, but also on the details of the electron capture properties of the stellar matter. Properties of nuclei at finite temperatures and density during this phase of the collapse, where shell and pairing corrections are relevant, are being computed [16.

The rate of generation of neutrinos per nucleon in a given energy band $E_{\nu}$ to $E_{\nu}+d E_{\nu}$ taking account of relative abundance of free protons and nuclei, is given by:

$$
d Y_{\nu}\left(E_{\nu}\right)=d \lambda_{f p}\left(E_{\nu}\right) X_{p}+d \lambda_{H}\left(E_{\nu}\right)\left(1-X_{n}-X_{p}\right) / A
$$

where A represents the Atomic weight of the ensemble of nuclei present in the core, taken here to be represented by a single "mean" nucleus as in earlier works [9]. The differential 
neutrino production rates for free protons and heavy nuclei are:

$$
d \lambda_{f p, H}=\frac{\log 2}{(f t)_{f p, H}} \frac{<G>}{\left(m_{e} c^{2}\right)^{5}} \frac{E_{\nu}^{2}\left(E_{\nu}+Q_{f p, H}\right) \sqrt{\left.\left(E_{\nu}+Q_{f p, H}\right)^{2}-\left(m_{e} c^{2}\right)^{2}\right)}}{\left(1+\exp \left(E_{\nu}+Q_{f p, H}-\mu_{e}\right)\right)} d E_{\nu}
$$

where the Coulomb correction factor $\langle G\rangle$ has been taken as $\approx 2$ for heavy nuclei and 1 for free protons. The Q-value, assuming that the strength is concentrated in a single state, is given as: $Q=\left(\hat{\mu}+1.297+E_{G T}\right)$ where $\hat{\mu}\left(=\mu_{n}-\mu_{p}\right)$ is the difference in the neutron and proton chemical potentials when free nucleons coexist with a distribution of neutron rich nuclei in nuclear statistical equilibrium, and $E_{G T}$ is the energy of the Gamow-Teller Resonance centroid. The centroids in fp-shell nuclei, found from experimental data from $(\mathrm{n}, \mathrm{p})$ reactions have been used for characterizing GT transitions in these nuclei $1 \mathrm{id}$. and are close to the value $(3 \mathrm{MeV})$ used here. The Fermi-energy $\mu_{e}=11.1\left(\rho_{10} Y_{e}\right)^{1 / 3} \mathrm{MeV}$. The difference in chemical potentials $\hat{\mu}$, and the relative fraction of free protons are obtained from a low density analytic equation of state similar to that in [9] with modifications noted in 8 .

We present a typical ("snapshot") spectrum of neutrinos from a $15 \mathrm{M}_{\odot}$ star's core collapse in Fig 1 within a narrow range of stellar core density around $10^{11} \mathrm{~g} / \mathrm{cm}^{3}$. Note the two separate peaks due to neutrinos from capture on free protons and heavy nuclei with clearly non-thermal spectrum. On the other hand, in Fig 2(a) we present a cumulative spectrum of neutrinos from the same star emitted till the stellar core density reaches $2.4 \times 10^{11} \mathrm{~g} / \mathrm{cm}^{3}$ (typically 120 milliseconds after the onset of collapse, and about 10 milliseconds before core bounce). The broader distribution of the neutrinos from captures on free protons reflects the full range of electron Fermi energies extant due to the variation of stellar core density as the collapse proceeds. Allowed capture rate on heavy nuclei is strongly attenuated, once the stellar core reaches a density of approximately $10^{11} \mathrm{~g} / \mathrm{cm}^{3}$ at which point the nuclei enter the neutron shell blocked phase. The range of density for which heavy nuclear capture effectively persists is therefore somewhat more restricted. As a result the cumulative spectrum shows a substantial fraction of high energy neutrinos. As the expected number of neutrinos detected depends strongly on the overall fraction of the high energy neutrinos from proton capture, some conditions of stellar collapse may be easier to detect than others. Figures 2(b) and 2 (c) gives the expected number of detections in the SNO and Super-Kamioka detectors, after folding the incident spectrum through the relevant interaction cross-sections assuming uniform and full detection efficiency. The spectra shown in Figs 1 and 2 are calculated with an effective sum-rule of the matrix element $\left|M_{G T}\right|^{2}=1.2$ and reduced only when $(\mathrm{A}, \mathrm{Z})={ }^{74} \mathrm{Ge}$ is reached. The former value for unblocked nuclei is characteristic of shell model calculations of $\beta$-strength function in iron isotopes [17]. We have also calculated spectra with $\left|M_{G T}\right|^{2}$ $=2.5$ in the unblocked era and found the cumulative spectra qualitatively similar, although somewhat deficient in higher energy neutrinos (results summarized in Table 1). The set of mean-nucleus transition matrix elements used for generating Figs. 2(a), (b) and (c) may be more realistic, because the A distribution of nuclei in collapsing stellar core is rather broad, and even at the onset of stellar collapse, the mean nucleus is fairly close to the neutron shell-blocked configuration. Therefore a substantial fraction of the nuclei present in the core may already have low capture transition strengths at the beginning of collapse. This fraction becomes larger after the mean nucleus enters the shell-blocked phase. Although the spectra have been obtained in the "mean nucleus" approximation while in reality the stellar 
core consists of an ensemble of nuclei, only a few nuclei with the largest abundance and the smallest effective Q-value (allowed) are expected to dominate the heavy capture spectrum (see e.g. [18]). Results of another example (core collapse of a $25 \mathrm{M}_{\odot}$ star) are summarized in Table 1 . This case had a higher core averaged entropy than the $15 \mathrm{M}_{\odot}$ case, and as a result has a larger fraction of free protons in the core. Consequently the cumulative spectrum is richer in high energy neutrinos.

To compare the numbers expected from the pre-trapping phase with the post core bounce phase, note that the number of neutrinos of all flavors in the post bounce phase from a SN $1 \mathrm{kpc}$ away is expected to be 592,000 in Super Kamioka (550,400 from $\bar{\nu}_{e}(p, n) e^{+}$and 41,600 from all types of neutrino electron scattering); similarly, 51,500 (neutrinos of all flavors and their anti particles) are expected in SNO (see eqs. 7.A.3, 7.A.4 and 7.A.7 of [四).

Neutrino spectroscopy of the final state of a star would be possible provided that the event occurs at a relatively close distance. Although, a priori, these may be rare events, there have been a number of historical Supernovae, as well as detected radio pulsars within a distance of about $2 \mathrm{kpc}$. There are a number of star forming regions nearby (such as the Orion complex - about 440 pc away), which are sites of potential supernova progenitor. A detection by underground neutrino experiments could constrain features of theoretical calculations of both collapse and explosion era of type II Supernovae as well as shed light on the characteristics of the stellar core.

This work was partially supported through the 8th Five Year Plan Project 8P-45 at Tata Institute. A.R. was supported by a National Research Council Senior Research Associateship at NASA/Goddard Space Flight Center. 


\section{REFERENCES}

[1] A. S. Burrows, in Supernovae, ed. A. G. Petschek, Springer-Verlag (1990)

[2] A. Ray, F. K. Sutaria and J. A. Sheikh, Proceedings of Workshop on "SN1987A: Ten Years Later" La Serena, Feb, 1997.

[3] W. D. Arnett, Astrophys. J. 218, 815 (1977)

[4] S. W. Bruenn, Astrophys J. Supl. 58, 771 (1985)

[5] H.-T. Janka, Proceedings of the Workshop on "SN1987A: Ten Years Later" - ed. M. Phillips et al. (1997)

[6] J. N. Bahcall, K. Kubodera and S. Nozawa, Phys. Rev. D 381030 (1988)

[7] A. Ray, S. M. Chitre and K. Kar, Astrophys. J. 285, 766 (1984).

[8] G. M. Fuller, Astrophys. J. 252, 741 (1982)

[9] H. A. Bethe, G. E. Brown, J. Applegate and J. M. Lattimer, Nucl. Phys. A324 487 (1979)

[10] F. K. Sutaria and A. Ray, Phy. Rev. C 52, 3460 (1995)

[11] Sudbury Neutrino Observatory Proposal, SNO-87-12, October (1987).

[12] L. M. Segal, Nucl. Phys. B70, 61 (1974)

[13] K. Kar and A. Ray, Phys Lett 96A, 322 (1983)

[14] A. Zaringhalam, Nucl. Phys. A404, 599 (1983)

[15] T. Weaver, S. E. Woosley, G. M. Fuller, Nuclear Astrophysics: Essays in honor of J. Wilson, ed. J. Centrella et al, 374 (1985).

[16] F.K. Sutaria, J.A. Sheikh and A. Ray, Nucl. Phys. A., Proceedings of "Nuclei in the Cosmos" $4^{\text {th }}$ International conference in Nuclear Astrophysics (1996) in the press.

[17] S.D. Bloom and G.M. Fuller, Nucl. Phys. A440, 511.

[18] J. Cooperstein and J. Wambach, Nucl. Phys. A420, 591 (1984). 


\section{TABLES}

TABLE I. Pre-trapping neutrino detections in SNO and Super Kamioka with hardness ratios up to $\rho_{10}=24.16$ for indicated heavy nuclear e-capture matrix elements for $15 \mathrm{M}_{\odot}$ Fuller (1982) and $25 \mathrm{M}_{\odot} \mathrm{WWF}$ presupernova stars.

\begin{tabular}{c|c|c|cc|cc|cc|}
\hline \hline Star Mass & $\left|M_{G T}\right|^{2}$ & $t_{\text {collapse }}$ & \multicolumn{2}{|c|}{ Pre-trapping Variables } & \multicolumn{2}{|c|}{ No. Detected } & \multicolumn{2}{|c}{ Hardness Ratio ${ }^{\dagger}$} \\
& & $(\mathrm{ms})$ & $Y_{\text {ef }}$ & $S_{f} / k_{B}$ & $\mathrm{SNO}$ & $\mathrm{S}-\mathrm{K}$ & $\mathrm{S} O$ & $\mathrm{~S}-\mathrm{K}$ \\
\multirow{3}{*}{$15 \mathrm{M}_{\odot}$} & $1.2 / 0.1$ & 120 & 0.3909 & 1.0021 & 82 & 394 & 0.2786 & 0.8540 \\
& $2.5 / 0.1$ & 120 & 0.3896 & 1.0085 & 66 & 344 & 0.2876 & 0.9537 \\
$25 \mathrm{M}_{\odot}$ & $1.2 / 0.1$ & 190 & 0.3828 & 1.1080 & 120 & 566 & 0.2878 & 0.8319 \\
& $2.5 / 0.1$ & 190 & 0.3813 & 1.1204 & 99 & 499 & 0.2916 & 0.9190 \\
\hline \hline
\end{tabular}

$\dagger$ The hardness ratio denotes the number of neutrino events in the $5 \mathrm{MeV} \leq E_{\nu_{e}} \leq 12 \mathrm{MeV}$ and $12 \mathrm{MeV} \leq E_{\nu_{e}} \leq 25 \mathrm{MeV}$ bands. 


\section{FIGURES}

FIG. 1. "Snapshot" neutrino fluence $\left(\mathrm{MeV}^{-1}\right.$ per baryon in a narrow density interval $\Delta \rho_{10}=0.0002$ around $\rho_{10}=9.8668 \mathrm{gmcm}^{-3}$ for a $15 \mathrm{M}_{\odot}$ star at $\mathrm{D}=1 \mathrm{kpc}$ and $\left|M_{G T}\right|^{2}=1.2$ and later 0.1 .

FIG. 2. (a) Cumulative neutrino fluence up to $\rho_{10}=24.16 \mathrm{gmcm}^{-3}$, with $\mathrm{M}=15 \mathrm{M}_{\odot}, \mathrm{D}=1$ kpc and $\left|M_{G T}\right|^{2}=1.2$ and later 0.1. (b) The spectrum in (a) folded with the detection cross-section

for c.c. reaction $\nu_{e}(d, p p) e^{-}$in SNO. (c) The spectrum in (a) folded with the detection cross-section for $\nu_{e}-e^{-}$scattering in Super Kamioka. 


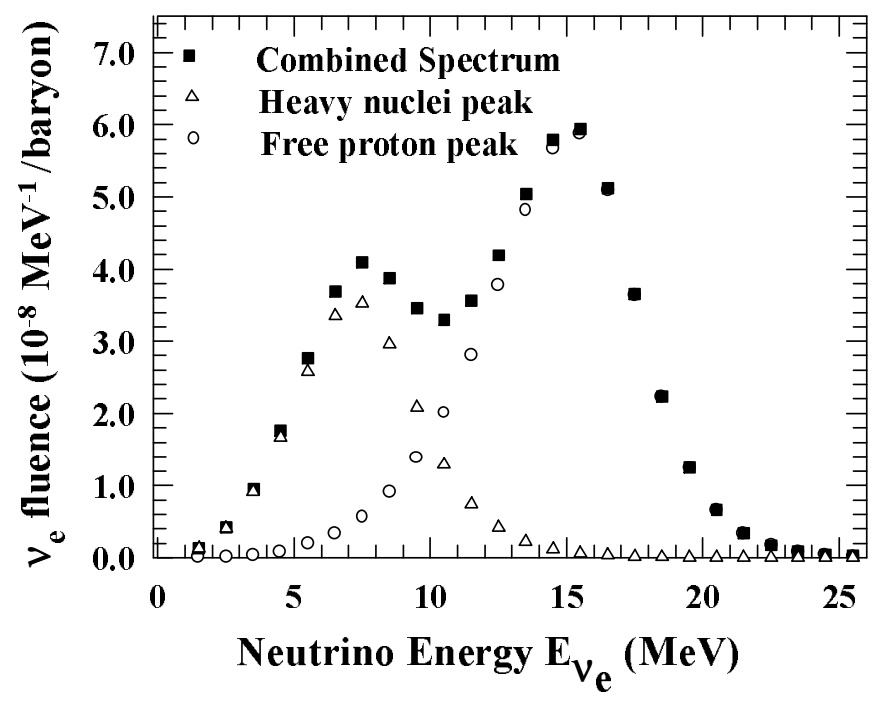




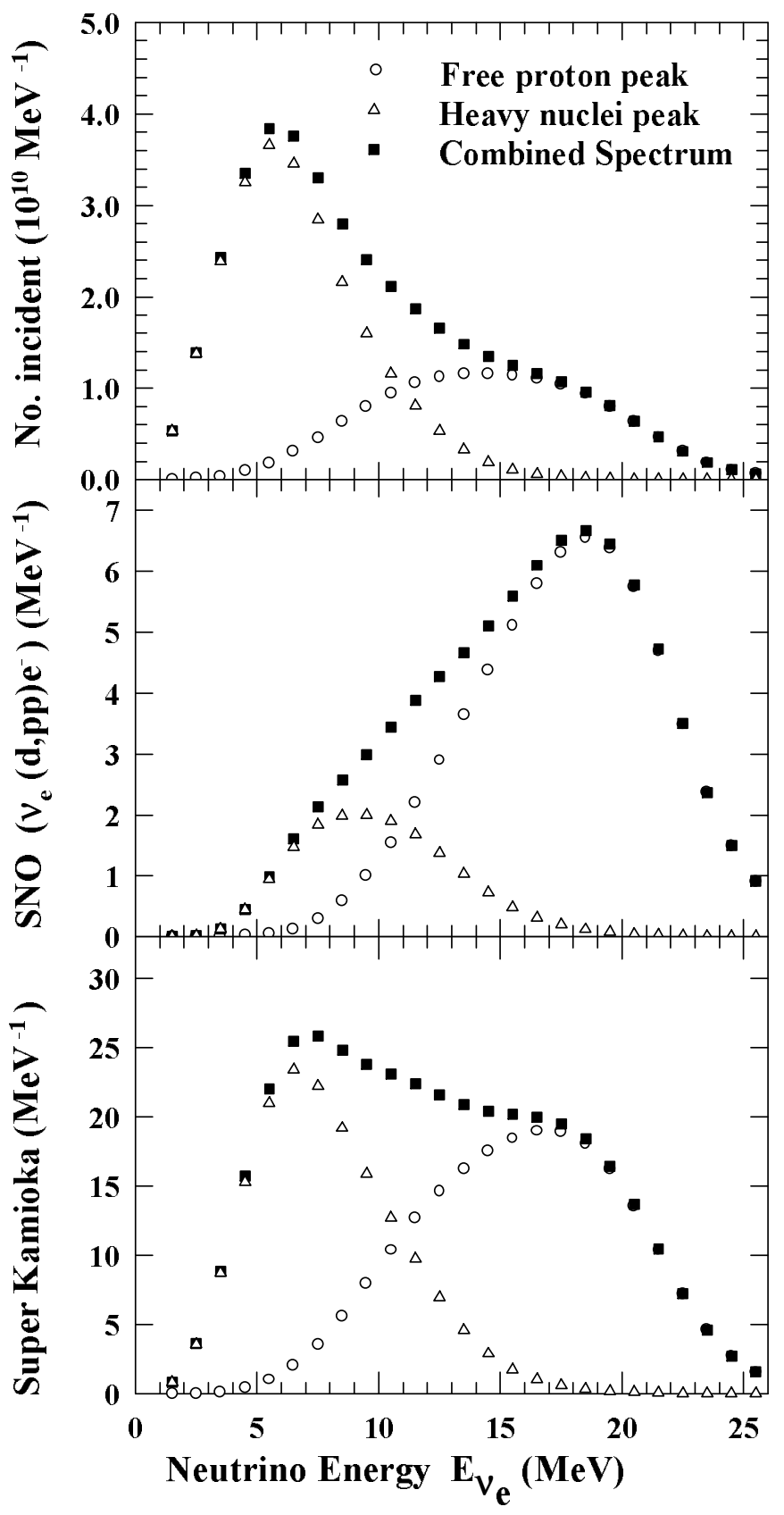

